\title{
Frontières
}

\section{Mort et deuil en contexte de dépendances}

\section{Amnon Jacob Suissa et Lucie Fréchette}

Volume 16, numéro 2, printemps 2004

Deuil, blessure vive

URI : https://id.erudit.org/iderudit/1074115ar

DOI : https://doi.org/10.7202/1074115ar

Aller au sommaire du numéro

Éditeur(s)

Université du Québec à Montréal

ISSN

1180-3479 (imprimé)

1916-0976 (numérique)

Découvrir la revue

Citer cet article

Suissa, A. J. \& Fréchette, L. (2004). Mort et deuil en contexte de dépendances. Frontières, 16(2), 43-49. https://doi.org/10.7202/1074115ar

\section{Résumé de l'article}

L'article explore le problème social des dépendances et amorce la réflexion sur le deuil qui s'ensuit dans la perspective de son impact sur les dynamiques familiales. Devant l'ampleur des formes de décès possibles dans le champ des dépendances, le texte traite d'abord la question à partir d'une lecture des perspectives de contrôle social pénal et thérapeutique. La deuxième partie décrit la façon dont les toxicomanies et les dépendances sont en rapport avec la mort et le deuil. Il analyse en troisième lieu en quoi le deuil des familles est particulièrement affecté par les décès en contexte de dépendances pour ensuite traiter des modes d'intervention en fonction de l'association dépendances-mort-deuil. 


\section{Résumé}

L'article explore le problème social des dépendances et amorce la réflexion sur le deuil qui s'ensuit dans la perspective de son impact sur les dynamiques familiales. Devant I'ampleur des formes de décès possibles dans le champ des dépendances, le texte traite d'abord la question à partir d'une lecture des perspectives de contrôle social pénal et thérapeutique. La deuxième partie décrit la façon dont les toxicomanies et les dépendances sont en rapport avec la mort et le deuil. Il analyse en troisième lieu en quoi le deuil des familles est particulièrement affecté par les décès en contexte de dépendances pour ensuite traiter des modes d'intervention en fonction de l'association dépendancesmort-deuil.

Mots clés : dépendances - deuilfamilles-intervention.

\section{Abstract}

The article explores the social issue of addiction and its impact on family dynamics when it invloves death and mourning. Given the wide range of possible deaths in the wake of addictions, the text first addresses the topic through an examination of the different perspectives relating to social, penal and therapeutic control. The second part sheds light on how drug abuse and addiction are related to death and grieving. In the third section, an analysis of how the grieving of families is particularly affected by deaths in a context of addiction and opens a discussion of intervention methods through a functional association of addiction death-grieving.

Key words: addictions - mourning family-intervention.

$\begin{array}{lllllllll}R & \text { E } & C & H & \text { E } & \text { R } & C & H & \text { E }\end{array}$

\section{Mort et deuil en contexte de dépendances}

\author{
Amnon Jacob Suissa, Ph.D., \\ professeur, Département de travail social \\ et des sciences sociales, \\ Université du Québec en Outaouais. \\ Lucie Fréchette, Ph.D., \\ professeure, Département de travail social \\ et des sciences sociales, \\ Université du Québec en Outaouais.
}

Les décès à la suite des dépendances: abus de psychotropes, jeu compulsif, workaholism, dépendance amoureuse, comportements à risque et/ou recherche de sensations fortes telles que les sports extrêmes ne sont pas rares. Les diverses formes de toxicomanie et les dépendances sont souvent rapportées dans la littérature comme des conduites à risque pouvant entraîner la mort (McCleary, 2002 ; Tousignant et Payette, 1997 ; Maris et al., 1992; Choquet, 1999). Curieusement, la littérature sur le deuil ne s'y réfère que rarement. Notre article veut explorer le problème social des dépendances et amorcer la réflexion sur le deuil qui s'ensuit dans la perspective de son impact sur les dynamiques familiales. Devant l'ampleur des diverses formes de décès possibles prévisibles ou non dans le champ des dépendances, nous traiterons d'abord la question à partir d'une lecture des perspectives de contrôle social pénal et thérapeutique. Dans une deuxième partie, nous identifierons la façon dont les toxicomanies et les dépendances sont en rapport avec la mort et le deuil. Nous étudierons ensuite en quoi le deuil des familles est particulièrement affecté par les décès en contexte de dépendances. Finalement, nous traiterons de divers modes d'intervention en fonction de l'association dépendancesmort-deuil.

\section{LES PERSPECTIVES PÉNALE ET THÉRAPEUTIQUE EN RÉPONSE AUX PROBLĖMES SOCIAUX LIÉS AUX DÉPENDANCES}

Au plan historique, il y a lieu de rappeler que les systèmes légaux de gestion sociale se sont constitués et développés en centrant leurs interventions, d'abord et avant tout, contre des populations considérées comme distantes socialement, voire déviantes. À ce titre, les indigents, les gitans, les pauvres et les esclaves illustrent bien cette réalité historique. Il en est de même aujourd'hui dans la gestion pénale des toxicomanes, des immigrants, des prostitués et des sans-abri. Parmi les perspectives de contrôle social les plus appliquées dans le champ des dépendances, on trouve celles de l'ordre pénal et thérapeutique / médical (Suissa, 1998).

Le recours au pénal se retrouve généralement centré dans une tentative de réduire la distance et le désengagement des citoyens dans leur rapport à l'ordre social élargi. Par 
réduction de la distance et le désengagement, il faut entendre le fait que des personnes ayant des liens sociaux considérés comme faibles seront plus assujetties à une réponse d'ordre pénal (Horwitz, 1990). Dans cette logique, un toxicomane socialement plus distant, à cause du contexte d'illégalité de certaines substances, par exemple, sera plus vulnérable à la réponse pénale. À l'opposé, un toxicomane qui possède des liens sociaux et familiaux forts, et donc considéré comme plus intégré sur le plan social, aura tendance à être moins assujetti à la réaction pénale et plus à la réaction thérapeutique et médicale. En d'autres termes, la capacité ou l'incapacité de créer des liens sociaux semble constituer un facteur prépondérant dans le processus décisionnel de prise en charge des comportements considérés comme déviants. Dans cette optique, le niveau de fonctionnement social au sein des réseaux familiaux, combiné avec la capacité de créer ou non des liens sociaux forts dans l'espace social élargi, constituent non seulement la pierre angulaire sur laquelle repose le type de réaction, mais également le choix à privilégier dans les mesures de contrôle.

La perspective thérapeutique, quant à elle, relève le phénomène de la médicalisation comme modalité de contrôle social, par laquelle on en vient à définir et à traiter les problèmes sociaux comme des problèmes médicaux (Conrad, 1995). Parmi les formes de déviance médicalisées, nous retrouvons les dépendances (toxicomanies, workaholism, dépendance affective, etc.), les troubles alimentaires et les activités compulsives, comme celles qui sont en lien avec les jeux de hasard et d'argent. Conrad rapporte que des facteurs historiques et sociaux ont favorisé l'apparition de la médicalisation comme mode de gestion des problèmes sociaux. Parmi ceux-ci, notons un déclin de la religion, une fois inébranlable dans la science, la rationalité et le progrès, le pouvoir et le prestige accrus de la profession médicale, une tendance humanitaire généralisée dans les sociétés occidentales. Aussi, le $\mathrm{XX}^{\mathrm{e}}$ siècle a été caractérisé, au plan historique, par une montée prééminente de l'institution médicale. En parallèle à cette ascendance, la profession médicale, en tant qu'institution, est devenue de plus en plus impliquée dans le contrôle de la déviance qui était alors sous l'égide de l'État ou de l'Église. C'est dans ce contexte qu'on peut mieux situer la question de la médicalisation des dépendances. Dans ce champ spécifique, le modèle traditionnel nordaméricain fondé sur le concept de la maladie soutient qu'il existe un phénomène unique identifiable comme étant une maladie permanente, irréversible et progressive qui se développe à travers des phases distinctes et identifiables. L'attrait exercé sur la population nord-américaine par ce modèle thérapeutique de la maladie peut s'expliquer principalement par une dé-stigmatisation sociale de l'abuseur (Breggin et Cohen, 1999 ; Tournier, 1985, p. 44).

À la lumière de ces observations, il est clair que la place sociale des personnes souffrant de toxicomanie, dans le sens des liens et des rapports sociaux, joue un rôle déterminant, non seulement dans le choix des trajectoires personnelles et familiales, mais également dans le choix des mesures de solutions à privilégier. Nous pouvons également constater que les formes et les styles de contrôle varieront selon les conceptions de la déviance des gens au pouvoir, la capacité du réseau primaire (milieu sociofamilial, amis) à tisser des liens sociaux et à résoudre les conflits, les modes d'intervention et les disciplines privilégiées dans la gestion sociale des personnes dépendantes. La question du décès en contexte de dépendances et de toxicomanies et celle du deuil n'échappent pas à l'influence du contexte pénal et médical entourant ces phénomènes dans notre société. On peut faire l'hypothèse que la stigmatisation de la famille endeuillée sera plus ou moins forte selon que le décès est plus ou moins associé à une perspective pénale ou médicale.

\section{TOXICOMANIES ET DÉPENDANCES. LEUR RAPPORT À LA MORT ET AU DEUIL}

\section{UNE ASSOCIATION MULTIFORME}

On ne peut que constater de multiples visages aux décès en contexte de toxicomanie ou de dépendance. Dans le champ des dépendances, les morts imprévisibles sont, toute proportion gardée, monnaie courante. On peut penser aux décès par abus (overdose), par sida et toxicomanie en contexte d'illégalité, par suicide, par le biais d'activités dans les milieux criminels, etc. (Hankins, 1997). Même si l'association toxicomanie-dépendance-mort est connue, la toxicomanie et les dépendances créent un certain flou quand elles sont reliées à la question de la mort. Leur lien causal avec la mort est d'intensité variable. D'une part, on entend par là que les toxicomanies ou les dépendances constituent des facteurs de risque affectant la santé au point de mettre en péril la vie de ceux qui s'y adonnent. Ainsi, le décès d'un patient atteint de cirrhose n'est pas étranger à une histoire personnelle marquée par de nombreuses années d'alcoolisme. D'autre part, on peut aussi attribuer aux dépendances la cause directe de décès. Les cas de mort par surdose en sont un exemple. En troisième lieu, les dépendances contribuent à placer les gens en situation où ils en viennent à poser des gestes léthaux ou susceptibles d'entraîner la mort. On retrouve ainsi plusieurs cas de suicides au sortir des casinos à la suite de pertes importantes au jeu (Suissa, 2004). Ou encore, on n'a qu'à consulter la nécrologie dans les journaux du lundi matin pour constater que la consommation excessive d'alcool constitue un facteur en présence dans des accidents de la route avec victimes. Finalement, certaines dépendances peuvent être associées au monde criminel et à des activités mortifères. Des luttes de territoires pour la vente de psychotropes illicites entraînent des décès, des vols ou des agressions commis pour financer la consommation de psychotropes peuvent mal tourner et faire des victimes.

La toxicomanie et les dépendances peuvent produire différentes catégories de victimes. Parmi celles-là, on retrouve d'abord les personnes dépendantes elles-mêmes comme dans les cas de décès à la suite de surdose, les suicides de dépendants aux jeux de hasard et d'argent. On retrouve ensuite des proches, le plus souvent des membres de la famille des personnes aux prises avec une dépendance. De jeunes enfants sont souvent négligés et maltraités gravement par des parents sous influence de psychotropes. Des conflits conjugaux chez des conjoints en état d'ébriété dégénèrent et donnent lieu à des comportements violents qui peuvent se révéler fatals. Finalement, la mort peut atteindre des gens sans aucune histoire de dépendance comme le dépanneur victime d'un vol ou des personnes victimes d'accidents de la route causés par des conducteurs en état d'ébriété.

L'association dépendances-mort est incomplète si on ne lui adjoint pas la composante deuil. En effet, les situations mortifères en lien avec les dépendances ne sont pas sans influence sur le processus de deuil et vice-versa. Bien sûr, les réactions de deuil ont certaines composantes que l'on pourrait qualifier d'universelles ou de fréquentes dans notre contexte culturel. Quand un contexte particulier entoure la mort, le deuil des proches est souvent plus complexe.

Nous illustrons une partie de nos propos à partir d'une histoire de suicide en lien avec la dépendance au jeu. Si le taux de suicide dans la population peut aller jusqu'à $3 \%$, celui lié au jeu compulsif chez les joueurs dits pathologiques peut atteindre entre 17 et $24 \%$ (Bailey, 2003). Les données préliminaires du Bureau du coroner en chef du Québec pour 2001 révèlent une variété impressionnante de formes de suicide associé au jeu compulsif. Les suicides par pendaison semblent être le choix premier pour la majorité des suicidés, suivis d'usage d'armes à feu, d'intoxication à la cocaïne, etc. Dans une étude intitulée Caractéristiques des suicides liés au jeu 
pathologique, Bourget, Gagné, Ward et Turmel (2000) révèlent qu'il n'y avait aucun signe avant-coureur dans $65 \%$ des cas, $12 \%$ avaient contacté un médecin et $20 \%$ avaient divulgué une intention suicidaire dans le mois précédant le geste. Des notes de suicide avaient été laissées dans 50\% des cas alors que l'intoxication à l'alcool était présente dans $25 \%$ des cas.

Les retombées de la dépendance au jeu sur les familles peut-être extrêmement sévère et tragique, car ce sont les proches directs qui doivent supporter cette dynamique de mensonge, de dénigrement, de stress financier et de souffrance généralisée. Parmi les conséquences néfastes répertoriées, Reno (2000) relève que les meurtres avec violence familiale en lien avec les problèmes de dépendance au jeu sont présents dans 11 des 51 États américains, que les enfants sont abandonnés dans l'enceinte de casinos, que $28 \%$ des couples divorceraient comme résultat direct des problèmes de dépendance au jeu, que les enfants décèdent parce que livrés à eux-mêmes dans les voitures des parcs de stationnement de casinos ou de maisons de jeu dans des conditions de grande chaleur. Le Québec voit son nombre de joueurs dépendants augmenter. On y remarque aussi que de plus en plus de jeunes s'adonnent aux jeux de hasard et d'argent. On ne peut qu'être sensible à l'association dépendance-mort-deuil. La recherche et l'observation clinique démontrent cependant que la dépendance à ce type particulier d'activités est dommageable (Suissa, 2003). La majeure partie des suicides associés au jeu compulsif, selon le Bureau du coroner en chef du Québec, confirme une certaine tendance à établir un lien entre les ALV (appareils de loterie vidéo) et l'incidence du suicide et le jeu. D'autres études sur les tentatives de suicide et les idéations suicidaires en lien avec le jeu compulsif reflètent ce même portrait (Petry et Kiluk, 2002 ; McCleary, Chew, Merrill et Napolitano, 2002).

C'est ce qui motive le choix que nous avons fait d'illustrer l'association dépendance-mort-deuil à partir d'un cas connu des milieux de recherche intéressés par les dépendances. La question est au cœur de débats scientifiques et cliniques au Québec en raison de la légalisation de formes de jeux de hasard et de loteries et avec l'apparition des premiers casinos en 1993.

La famille $\mathrm{X}$ vivait une vie considérée harmonieuse et confortable au plan financier. À l'âge de 17 ans le fils a été initié par d'autres jeunes aux machines et aux jeux d'arcades. La mère voyait alors cette activité comme totalement inoffensive et bénigne ne l'associant au grand jamais à une activité pouvant provoquer la mort. Le jeune X n'était pas dysfonctionnel et n'avait jamais présenté de tendances suicidaires. Les parents ont toutefois remarqué une diminution de ses résultats scolaires, un ralentissement de ses relations sociales et le fait qu'il semblait parfois aux prises avec des problèmes financiers. Les parents affirment avoir réagi en luttant contre cette tendance au jeu et en demandant de l'aide à Gamblers Anonymes. Après le décès, il ne semble pas à la mère que l'organisme les ait prévenus du fait que l'accessibilité aux jeux de hasard pouvait mettre le jeune en danger même de mort et son impression est que le jeune était seulement invité à se responsabiliser.

Durant l'année précédant son décès, $\mathrm{X}$ affichait des signes de désespoir liés à sa dépendance aux AVL. Une entrevue avec sa mère laisse entendre qu'il avait intériorisé des sentiments d'impuissance, d'inutilité et de dépression. Il est entré dans ce cycle infernal parce qu'il avait honte de la condition psychosociale dans laquelle il était «tombé». La situation du jeune était en effet grave. La dépendance lui a hasard et d'argent. Des membres de la famille étendue avaient aussi de la difficulté à composer avec le suicide.

Le contexte de dépendance du décès n'est pas sans influence sur le processus de deuil de la famille. Le père a vécu un choc aigu et s'est senti envahi par le sentiment d'impuissance qui l'habitait depuis longtemps en fonction du problème de son fils. Il s'est investi du rôle de protection de sa conjointe et de sa fille qui l'a aidé à traverser l'épreuve. Rôle qui l'a mené à verser dans la surprotection de la jeune fille. La famille s'est sentie seule et désemparée et ne comprenait pas vraiment ce qui avait pu se passer. La mère dit: «ça m'a pris plus de deux ans pour en savoir un peu plus sur ce phénomène et à traverser les chemins de souffrance liés à la stigmatisation, au choc, à l'incompréhension, à l'absence de support...». Le deuil étant complexe et la famille ne se sentant pas soutenue, celle-ci s'engage dans une thérapie familiale de six mois, thérapie axée sur la résolution de deuil. La mère y ajoute un suivi en psychiatrie durant une année supplémentaire.

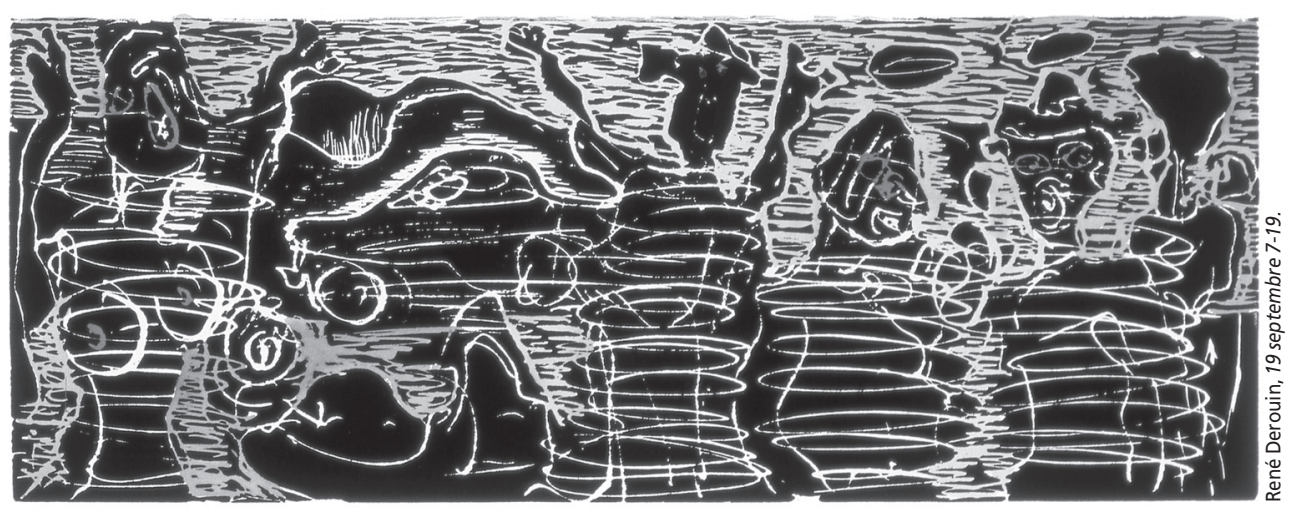

coûté $100000 \$$. Au moment de sa mort, il avait contracté $10000 \$$ de dettes et trois mois d'arriérés de paiement pour sa voiture. C'est dans ce contexte de grand endettement que $X$, six semaines avant son $26^{\mathrm{e}}$ anniversaire de naissance, s'est enlevé la vie dans le garage de la résidence familiale.

La famille a vécu un deuil difficile. Le suicide n'a pas fait l'objet de tabou. Il a cependant fait partie du paysage de deuil de l'ensemble de la famille. Les parents et leur autre enfant, une fille âgée de sept ans à l'époque, ont souffert des réactions sociales stigmatisantes de l'entourage. La jeune fille rapporte qu'on la connaissait comme la sœur du suicidé aux jeux de
L'une des avenues qui a permis un travail de deuil a été l'engagement des parents dans une cause sociale pour contrer la progression des dépendances au plan social. "Ce qui m'a sauvé de ma souffrance, dit-elle, c'est mon implication sociale et mon travail de représentation sociopolitique. » En s'impliquant et en revendiquant la co-responsabilité de l'État dans la production des suicides en lien avec les jeux de hasard, et du décès de son fils en particulier, Mme P.V. est devenue, au fil des ans, une personnalité qui cristallise les paradoxes et certains effets psychosociaux pervers de la légalisation des jeux de hasard au Québec et au Canada. Madame donne des 
conférences dans les écoles de travail social, dans les regroupements sociaux divers et participe activement dans les congrès au plan national et international pour dénoncer le silence autour des tragédies associées au jeu, ce qu'elle nomme «la grande épidémie silencieuse». Son engagement a aussi eu des effets bénéfiques sur sa famille. Celle-ci est informée, comprend mieux le contexte qui a mené le fils au suicide et vit une plus grande transparence de communication intrafamiliale.

À la suite de cet encart, il est à propos de souligner que le deuil est bien documenté dans la littérature scientifique et la littérature d'intervention ou clinique, que l'on parle de réactions de deuil, de construction sociale du deuil, d'états de deuil ou d'étapes de deuil (Parkes, 1972 ; Flatt, 1987 ; Rosenblatt, 1988 ; Bacqué et Hanus, 2000 ; Séguin et Fréchette, 1999). On ne peut référer à tous les auteurs sur le sujet. Ils sont nombreux. À la lumière des travaux sur le deuil, on réalise qu'étudier les réactions de deuil exige d'analyser les éléments qui contribuent à la souffrance et exige de prendre en compte le contexte social en présence. La littérature fait aussi référence à des deuils marqués de caractéristiques particulières, le décès en contexte périnatal ou en début de vie (Mongeau, 2002; Fréchette, 1997 ; Dyregrov et Mathiessen, 1987), le deuil après un suicide (Séguin et al., 1994), celui des décès plus ou moins acceptés socialement (Doka, 1989), le deuil lors de décès à caractère violent (Weinberg, 1994 ; Malmquist, 1986) et d'autres. À propos du deuil à la suite d'un décès en contexte de dépendance, la littérature se fait plus discrète. Nos travaux antérieurs indiquent cependant que lorsqu'un contexte particulier s'ajoute à la souffrance des endeuillés, il importe d'identifier les éléments qui modifient la souffrance et l'expression $\mathrm{du}$ deuil des personnes ou des familles affectées. Nous nous y attardons dans la suite de cet article en référant principalement à trois éléments affectant le deuil : le deuil quand le décès est de caractère ambigü, le décès en situation d'illégalité et le décès empreint d'une tolérance sociale qui occulte la réaction des endeuillés.

\section{L'AMBIGUÏTÉ QUI BROUILLE}

LE PROCESSUS DE DEUIL

Quand on dit d'un décès qu'il est ambigu, l'expression renvoie le plus souvent à l'incertitude ou à la méconnaissance de prime abord de la cause ou de l'élément déclencheur du geste ou de la situation ayant causé un décès. Il peut aussi renvoyer au caractère confus ou inconnu des circonstances entourant un décès. Ainsi, un décès associé à la toxicomanie en contexte ou non de délinquance avec enquête de coroner laisse planer le doute dans l'esprit des familles endeuillées. Le corps de la personne décédée sera objet d'autopsie avant d'être rendu à la famille. Lorsqu'il y a enquête, le rapport est généralement disponible plusieurs mois après le décès. Selon Boss (1999), cette affliction fait vivre aux proches des sentiments de grande impuissance, d'anxiété et même de dépression. La confusion qui règne autour du décès et de ses causes ne placent pas les endeuillés proches en situation propice pour donner du sens à leur perte.

Comme c'est le cas pour tous les décès, un état de choc chez les proches suit l'annonce du décès. Dans les cas où une dépendance est associée au décès, l'incertitude s'ajoute bien vite aux autres états de deuil. On s'interroge alors plus sur le contexte dans lequel s'est produit le décès, sur la cause réelle du décès, sur la façon dont le décédé a vécu sa mort, etc. On se demande s'il a souffert, s'il a vu venir sa mort, s'il était en état de réagir, s'il souhaitait ou non mourir, s'il a cherché de l'aide, etc. Cette situation peut être encore aggravée quand parfois le corps de la victime n'est pas retrouvé ou quand on soupçonne que la mort est le résultat d'un acte criminel. Dans ces situations, depuis la perte par surdose jusqu'à l'acte criminel, le désarroi s'insinue chez les membres des familles et perturbe ou retarde la mise en œuvre des rituels qui accompagnent la mort.

Une des réactions adoptées pour transiger avec l'ambiguïté est celle du déni comme mécanisme premier de défense. Il sert alors de fonction adaptative de protection des personnes endeuillées contre la "pire des pensées» envers l'être cher disparu. Ne pas connaître, ou dénier les hypothèses intolérables associées à la mort, permettent, jusqu'à un certain point, aux survivants de garder un peu d'espoir.

Le caractère ambigu d'un décès n'a pas que des répercussions dans la famille immédiate. Il engendre souvent un questionnement dans l'entourage élargi de la personne décédée. En l'absence d'informations sur la cause ou les circonstances d'un décès, les hypothèses émergent bien rapidement dans l'esprit des uns et des autres dans le milieu de travail, dans les cercles fréquentés par le décédé, dans les milieux où évoluent des membres de sa famille, ou encore dans le voisinage.

\section{LE CONTEXTE D'ILLÉGALITÉ}

QUI PERTURBE LE PROCESSUS DE DEUIL

Le décès en contexte d'illégalité renvoie à des morts dans des circonstances où la loi a été enfreinte. Là encore, l'éventail des possibilités est large. Il peut s'agir de situations où la victime elle-même transgressait la loi ou de situations où quelqu'un décède parce qu'une autre personne transgresse la loi.

Les cas de décès en situations d'illégalité rendent eux aussi le deuil plus complexe. Les décès associés à la consommation d'héroïne ou encore les meurtres associés au trafic de psychotropes sont des exemples de situations qui posent un défi additionnel à la résolution du deuil. Dans ces circonstances, les réactions de deuil varieront selon que l'activité illicite de la personne était connue ou non de son entourage, selon que cette activité était acceptée, tolérée ou réprouvée par la famille, les proches ou l'entourage, selon que le décès était prévisible ou non en raison de la nature de la situation avec laquelle était aux prises la personne décédée. Dans tous les cas, le deuil est plus complexe. Nous concentrons notre propos sur trois états qui font habituellement partie du processus de deuil, la colère, la culpabilité et le sentiment d'impuissance (Jacques, 1998) et qui sont affectés de façon particulière lors de décès en contexte de dépendances.

Il n'est pas rare que la colère fasse partie des états de deuil. Même dans des cas de décès prévisibles chez des gens atteints de maladies à issue fatale, il arrive que les endeuillés éprouvent de la colère. Ils en veulent à l'un ou l'autre des membres de la famille de ne pas avoir suffisamment pris soin du défunt; ils en veulent au système de santé de ne pas avoir pu prolonger la vie du malade ; ils en veulent à Dieu d'avoir infligé ce malheur à leur famille. À plus forte raison, quand le décès se produit en dehors de ce qui est considéré la normalité, on peut s'attendre à ce que la colère fasse intensément partie des états de deuil des proches du décédé.

Cette colère peut être dirigée vers la personne décédée elle-même. Les familles en veulent à la personne de s'être engagée dans le problème qui a entraîné sa mort. La colère peut aussi être dirigée vers un proche, une relation ou un milieu qui à leurs yeux sont à l'origine du problème ou de la situation létale. Ainsi, on en veut à un frère, une sœur ou au cercle d'amis qui ont initié une personne à la consommation de psychotropes. On en veut au système scolaire qui n'a pas su contrôler la vente de psychotropes dans les cours d'école. On en veut à l'ex-conjoint qui a quitté le décédé, geste perçu l'ayant précipité vers la consommation d'alcool ou de psychotropes. La colère a besoin de s'exprimer. Il est parfois compliqué de le faire sans trop égratigner les proches. Quand cette colère est dirigée vers des membres de la famille, elle devient de plus un frein à l'expression du deuil dans la famille et au recours au soutien mutuel pour s'en sortir. 
Il arrive que les personnes décédées ne soient impliquées en rien dans des gestes illégaux mais soient tout simplement d'innocentes victimes de situations où il $\mathrm{y}$ a eu infraction à la loi. La colère fait alors partie du paysage des endeuillés. Elle est dirigée vers les personnes ayant causé le décès, qu'il s'agisse d'un conducteur ivre au volant, d'un agresseur avide d'argent pour satisfaire une dépendance. La colère peut alors être doublée du désir de vengeance (Weinberg, 1994). Dans les cas où l'agresseur n'est pas identifié, la colère mobilise les énergies autour de la recherche du coupable. La colère devient parfois haine et peut aller jusqu'à induire une tendance à l'agressivité. Les cicatrices ouvertes par ces états de deuil sont profondes et longues à refermer.

Le sentiment de culpabilité est lui aussi un état de deuil courant (Miles et Demi, 1986; Stroebe, 1987). Son intensité et sa durée sont toutefois variables selon les circonstances entourant le décès et selon la nature de la relation antérieure entre les endeuillés et le décédé. Les décès en contexte d'illégalité ravive cependant le sentiment de culpabilité souvent de façon intense. Un décès par surdose, un décès dans le monde interlope ou de la prostitution pose un problème aux proches qui s'en veulent de n'avoir pas pu le prévenir. La culpabilité est le lot des parents qui se demandent ce qu'ils ont fait pour que leur enfant en vienne à vivre dans des circonstances troubles. La culpabilité affecte les parents et les proches qui s'en veulent de n'avoir pas su évaluer la gravité d'une situation. La culpabilité mine ceux qui se taisaient ou feignaient d'ignorer la situation du décédé.

Dans un autre ordre d'idées, il faut souligner que le traitement médiatique de certains décès exacerbe souvent la culpabilité des proches des victimes et lui assortit un sentiment de honte en affichant au grand jour le contexte d'illégalité en cause dans le décès (Fréchette, 1988) La négligence d'un parent alcoolique est révélée au grand jour quand un enfant se noie dans la piscine familiale. Les démêlés avec la justice d'une jeune sont rendus publics quand elle est retrouvée sans vie dans une maison de prostitution. Autant de situations troubles dont l'exposition accentue le sentiment de culpabilité et la honte qui les accompagnent souvent.

Finalement, le sentiment d'impuissance transparaît aussi dans les réactions de deuil en contexte de dépendance. Bien souvent, les problèmes vécus par les gens décédés dépassaient la capacité d'agir des proches. Par exemple, la transaction avec une personne héroïnomane est fort complexe. Le parent, le frère ou l'ami ne peuvent s'improviser thérapeutes ni espérer sortir de force la personne de sa dépendance. Bien que les membres de la famille aient souvent échangé sur le sujet, ils se sentent impuissants et parfois même partie prenante du cycle de la dépendance. Parfois, ils ont pu implorer la personne dépendante de se faire traiter. Dans d'autres cas, des parents ont eu recours aux services de la Protection de la jeunesse ou à un centre d'accueil, espérant ainsi induire des changements chez un jeune engagé dans des comportements délinquants. Ces gens ont la conviction d'avoir essayé... mais en vain. Le sentiment d'impuissance s'est alors immiscé dans leur vie. Le décès du proche aux prises avec une dépendance ou des comportements déviants et délinquants qui lui sont associés ravive ce sentiment d'impuissance. Ils sont précipités dans l'impuissance ultime celle qui ne peut prévenir la mort de quelqu'un que l'on aime. Celle qui suit le suicide d'un endetté du jeu, celle qui suit la mort d'un toxicomane, celle qui suit la perte d'un enfant publiquement connu comme criminel.

\section{LA TOLÉRANCE SOCIALE QUI MET}

EN VEILLEUSE DES SOUFFRANCES TUES

Les décès en contexte d'illégalité ou ceux qui sont associés à la perspective pénale comme mode de contrôle social des dépendances auront tendance à être gérés différemment et jugés plus sévèrement. Un décès dit «classique» par alcoolisme ou tabagisme pour un père de famille comme cela se passe pour des milliers de familles en Amérique du Nord et ailleurs, par exemple, continuera à être l'objet encore aujourd'hui d'une plus grande acceptation sociale, car ces substances, étant plus intégrées à nos valeurs sociales et à notre culture, susciteront des réactions moins sévères. Par ailleurs, un décès impliquant alcoolisme et accident automobile avec facultés affaiblies aura tendance à être jugé plus sévèrement, car l'accident est géré et inscrit dans le biais criminel et pénal. Bien sûr, les membres de la famille impliquée dans ce type de tragédie vivront ouvertement des tensions graves, non seulement à cause de la perte de l'être cher, mais aussi à cause du jugement social plus sévère face au décès. Quand le décès relève d'une situation de dépendance socialement plus tolérée comme l'alcoolisme, on s'imagine cependant à tort que le deuil des proches n'en est pas perturbé.

Au plan clinique et conjugal, l'acceptation sociale du comportement de dépendance comme une maladie permet l'installation d'une dyade, où la responsabilité d'un partenaire dépendant est généralement évacuée à l'extérieur de la dynamique du couple, maladie oblige. La réalité du «je suis malade, ce n'est pas de ma faute » favorise le plus souvent chez la conjointe un rôle où elle doit généralement couvrir les problèmes vécus par son conjoint. Cette adaptation secondaire de la co-dépendante engendre une relation où la symétrie (relation fondée plus sur un rapport égalitaire) est bannie du champ de la réalité, alors qu'elle est la pierre angulaire de la confrontation et du changement individuel et social (Suissa, 2000). On entre alors dans une relation de type complémentaire et inégal, ce qui explique d'ailleurs la durabilité impressionnante des systèmes familiaux avec dépendances qui peuvent durer dix, vingt et trente ans. Ainsi compris, les gestes posés par la conjointe pour aider son conjoint dépendant considéré malade protègent non pas le dépendant mais le système de dépendance comme tel.

La nature de la relation établie entre la substance ou l'activité créant la dépendance et les membres d'une famille est déterminante de la façon dont la famille transige avec le problème d'un des siens (Weil, 1983). Le problème de dépendance est souvent présenté comme un problème individuel propre à la personne souffrante (Bennett, Steinglass, Wolin et Reiss, 2000 ; Figiel, 1988). Or, il y a lieu également de se tourner vers le système familial dans la mesure où il a permis de nourrir, jusqu'à un certain point, l'apparition du symptôme et l'installation de cette dépendance (Elkaïm, 1999; Ausloos, 1995) ou encore dans la mesure où tous les membres de la famille sont affectés par le problème vécu par l'un des siens. En effet, les familles souffrent des conséquences de l'alcoolisme d'un des leurs, conséquences affectives, sociales, économiques, etc. Cette souffrance fera partie de la toile de fond sur laquelle se développent les états de deuil quand vient à mourir la personne qui en est perçue comme responsable.

La relation ambivalente antérieure à la mort ravive généralement chez les endeuillés la tension chagrin-hostilité, nostalgie-haine, et l'entrée en deuil ferme la possibilité d'exprimer les reproches que l'on aurait toujours voulu adresser au décédé. Le deuil peut se transformer en deuil inhibé en ce sens que les manifestations de deuil sont mises en retenue, ne s'expriment pas ou ne sont pas apparentes. L'endeuillé cherche à signifier ainsi que la perte ne l'affecte pas vraiment même si ce n'est pas le cas. Ces deuils retenus constituent souvent des deuils différés. Les réactions comprimées en viendront à prendre le dessus lors de situations ou de paroles réactivant le traumatisme ou une souffrance tue. Le deuil dans les pertes de relations ambivalentes peut aussi se transformer en deuil élargi. L'endeuillé n'a pas l'impression d'avoir perdu une personne aimée qui lui 
rendait son affection mais a l'impression d'avoir perdu à tout jamais la possibilité d'être aimé ou de vivre une relation satisfaisante avec la personne disparue. Ainsi un enfant qui a perdu une mère cocaïnomane sera endeuillé non seulement de la perte de la personne, mais encore de la perte de la possibilité de retrouver un jour une relation de filiation maternelle acceptable. Son deuil couve un chagrin qui traduit son sentiment qu'il n'a jamais vraiment eu de mère.

En présence de la toxicomanie ou de l'alcoolisme, certains types de familles auront tendance à se replier sur elles-mêmes et développeront un système familial fermé. Les frontières externes dans ce type de familles seront généralement fermées pour ne pas dévoiler le secret familial et ne pas exposer ainsi les membres à un éclatement familial potentiel. Les liens sociaux sont plus rares et plus faibles, ce qui fait que le soutien se fait aussi rare au moment d'un décès. L'isolement familial s'ajoute au secret familial, ce qui bâillonne l'expression de la colère sous-jacente au deuil. Sous cet angle, la question des liens sociaux forts ou faibles des familles qui traversent l'épreuve de la vie avec un proche dépendant semble démontrer que les familles ayant un réseau primaire de liens sociaux forts seront plus aptes à traverser cette période de souffrance avec une plus grande résilience que celles ayant des liens sociaux faibles ou inexistants (Suissa, 2001). Ces liens seront aussi mis à contribution pour traverser l'épreuve de la mort. Leur force se révélera lénifiante si la communication est saine et si la famille n'est pas cimentée par le partage d'un secret concernant la dépendance du décédé.

\section{INTERVENIR DANS}

\section{DES SPHÈRES DIVERSIFIÉES}

L'intervention en fonction de la mort associée aux dépendances et toxicomanies peut s'adresser aux familles endeuillées pour favoriser l'expression la plus saine possible de la souffrance et aider à passer à travers le travail de deuil qu'affrontent ces familles. L'intervention et la relation d'aide auprès des endeuillés procèdent par cercles concentriques plus ou moins proches des endeuillés. On peut parler de soutien au sens large et formel par l'intermédiaire des rites sociaux ou religieux entourant la mort. Le soutien peut aussi prendre la forme de groupe d'entraide ou de groupes de soutien ou la forme d'aide individuelle ou de thérapie. Finalement, l'aide aux endeuillés s'opère aussi dans la sphère privée ou intime grâce au soutien des proches.

En ce qui concerne les rites et rituels entourant la mort, de tout temps, les cultures entretiennent des cérémonies et des pratiques visant à transiger avec ceux qui décèdent. Les rituels représentent des activités de convention sociale qui, selon Kollar (1989), comportent quatre buts dans les décès dits "conventionnels". Un but physique visant à faire face aux besoins biologiques proprement dits, un but social pour fournir un groupe de support pour les endeuillés dans l'installation d'une nouvelle dynamique familiale, un but psychologique qui renforce la réalité incontournable de la mort et enfin un but religieux ou spirituel qui offre une vision de la vie et de la mort par le biais des actes rituels. Ces divers buts constituent des composantes critiques pour traverser la souffrance et introduire les individus et leurs familles dans un processus de guérison face à la perte (Beder, 2002). Les réactions des groupes sociaux concernés et la reconnaissance sociale associée aux rituels funéraires seront altérées par des facteurs comme la nature de certains décès, les acteurs en présence et les normes et codes de conduite en vigueur dans le groupe et la société en question, etc. Ces facteurs, combinés à l'anticipation de la réaction sociale au type de décès avec lequel une famille transige, influeront sur les trajectoires qu'emprunteront alors les familles et leurs réseaux dans la prise en charge du décès en question et dans le recours à une ritualisation du passage de la vie à la mort. Dit autrement, le processus du décès sera intimement lié au degré de désirabilité sociale du type de décès en question qui à son tour mettra en route le processus de réaction sociale impliquant alors un jugement social.

Lorsque le décès est attribué à une dépendance au travail (workaholism / burnout) ou au tabagisme, la famille peut s'engager dans un processus de deuil socialement acceptable, voire désirable; par ailleurs, lorsque le décès se produit dans des conditions de dépendance en contexte de délinquance / déviance / criminalité ou dans une situation ambiguë, les survivants traverseront des périodes de souffrance plus intenses, plus longues avec des effets négatifs majeurs sur le développement des enfants dans ce type de familles (Lynley, 2001). Il n'est d'ailleurs pas dit que les situations socialement plus tolérées ne cachent pas elles aussi des deuils complexes. Dans plusieurs cas de deuil en lien avec des dépendances, le recours à l'intervention professionnelle ou à la relation d'aide est bénéfique.

En ce qui concerne la relation d'aide dans les cas où un contexte particulier s'ajoute à la souffrance des endeuillés, on devrait prendre en compte quatre éléments : ce qui amplifie la souffrance, le contexte social en présence, les modalités d'expressions du deuil, les adjuvants au travail $d u$ deuil. L'intervenant identifie d'abord en quoi la situation, dans le cas présentant une toxicomanie ou une dépendance, modifie la souffrance de la personne endeuillée. Par exemple, dans les cas de décès avec violence, le sentiment de vengeance peut dominer les états de deuil. Il cherche ensuite à connaître le contexte social dans lequel se vit le deuil. Il se demande 1) si la perte est reconnue socialement ; 2) si l'entourage est aidant ; 3) s'il y a des facteurs entravant le deuil comme une enquête de police, une exposition de l'intimité familiale dans les médias, des dynamiques familiales antérieures conflictuelles, etc. L'intervenant analyse ensuite en quoi les modes d'expression du deuil sont affectés par le contexte particulier. Un parent dont le deuil est soumis à un secret de famille, un conjoint qui nie le suicide d'un jeune joueur, la colère enfin permise envers un défunt parent alcoolique abuseur. Finalement, il s'interrogera sur les moyens les plus pertinents pour aider les endeuillés et l'on identifiera les formes de soutien requises et leur disponibilité dans l'environnement. Il ne faut alors pas oublier le recours à des ressources communautaires comme les centres de détresse, les lignes d'écoute téléphonique, les groupes d'entraide pour endeuillés et spécialement ceux qui portent une attention spéciale aux dépendances, même s'ils se font rares.

Dans un autre ordre d'idées, des endeuillés ou des regroupements de familles s'engagent dans des activités sociales ou politiques qui deviennent des adjuvants au travail de deuil. Ces gens affrontent leur souffrance en étant proactifs au plan social et politique. Ils militent en faveur de changements sociaux pour prévenir les dépendances ou encore deviennent bénévoles dans des associations venant en aide aux personnes aux prises avec le problème qui affectait l'être perdu. Dans le cas des décès impliquant l'alcoolisme, MADD (Mothers Against Driving and Drinking) représente un bel exemple de revendication d'une justice plus équitable où des milliers de familles s'organisent pour exiger des sanctions plus sévères. Depuis son positionnement comme acteur social et politique dans l'arène publique ces dernières années, ce groupe social de pression détient une balance de pouvoir non négligeable dans la gestion des modalités sociales et légales à appliquer. On peut également observer que cette implication activiste (advocacy), qui va jusqu'aux cours de justice, semble avoir une influence positive pour les familles des survivants dans la mesure où elles ne sont plus isolées avec leur détresse et qu'elles peuvent ainsi se projeter dans une trajectoire active de partage, de solidarité face à l'adversité.

Comme nous avons pu le constater au long de cet article, l'association dépendance- 
mort-deuil recouvre une grande diversité de situations prévisibles ou non, apparentes ou dans l'ombre, socialement acceptées ou non. La souffrance est cependant toujours de la partie autant au cours de la vie des familles dont l'un des leurs est aux prises avec une dépendance qu'au moment de la mort d'un membre de la famille. Le deuil qui s'ensuit participe d'une part du processus connu de deuil et d'autre part est sous influence de facteurs qui rendent son expression et sa résolution fort complexe. L'aide qui est alors disponible dans l'entourage se fait parfois plus rare ou moins adaptée aux situations associées aux dépendances. Quand les intervenants auprès des endeuillés seront mieux formés en rapport avec le phénomène multifactoriel des dépendances, ils seront mieux outillés pour accompagner les endeuillés affrontant un deuil issu de l'association dépendancemort-deuil. Finalement, cette souffrance des endeuillés ne doit pas seulement faire l'objet de l'aide au sein des cabinets de médecins, de psychologues ou de travailleurs sociaux. Elle doit inciter à la prise de parole et à l'action pour que les milieux de la santé, de la justice et des services sociaux dénoncent les causes sociales des dépendances et militent en faveur de législations et de choix de société qui contrent les facteurs favorisant le développement des dépendances. Briser l'association dépendance-mort-deuil ne relève pas que de la compassion. Il faut lui adjoindre la prévention.

\section{Bibliographie}

AUSLOOS, G. (1995). La compétence des familles, France, Éditions Érès, 173 p.

BACQUÉ, M.F. et M. HANUS (2000). Le deuil, Presses universitaires de France, coll. "Que sais-je ? », Paris, 7 p.

BAILEY, S. (2003). « Suicides, gambling link probed. Coroners and medical examiners will look at ways to track suicides tied to gambling », Newscan, vol. 5, $\mathrm{n}^{\circ}$ 22, 30 mai.

BEDER, J. (2002). "Mourning the unfound: How we can help », Families in Society: The Journal of Contemporary Human Services, vol. 83, n 4, p. 400-403.

BENNETT, L.A., P. STEINGLASS, S.J. WOLIN et D. REISS (2000). Alcoholic family. The first-lenght scientific exploration of the children of alcoholics, USA, Alibris.

BOSS, P. (1999). Ambiguous Loss, Cambridge, MA, Harvard University Press, 155 p.

BOURGET, D., P. GAGNÉ, H. WARD et S. TURMEL (2000). Caractéristiques des suicides liés au jeu pathologique, Bureau du coroner du Québec, Gouvernement du Québec, Canada.

BREGGIN, P. et D. COHEN (1999). Your Drug May Be Your Problem, Cambridge, MA, Perseus Books, 272 p.

CHOQUET, M. (1999). « Suicide à l'adolescence en France », Frontières, vol. 12, n 1, p. 54-61.
CONRAD, P. (1995). "Médicalisation et contrôle social ", Médicalisation et contrôle social, ACFAS, vol. 84, p. 9-31.

DYREGROV, A. ET S.B. MATHIESSEN (1987). «Stillbirth, neonatal death and sudden infant death (SIDS) : Parental reactions », Scandinavian Journal of Psychology, vol. 28, $\mathrm{n}^{\circ} 2$, p. 104-114.

DOKA, K.J. (1989). Disenfranchised grief, Toronto, Lexington Books, 347 p.

ELKAÏM, M. (1999). La thérapie familiale en changement, France, Le Plessis-Robinson, coll. "Les empêcheurs de penser en rond", $226 \mathrm{p}$.

FIGIEL, C. (1988). « Toxicomanie et environnement non familial : travail avec les réseaux de toxicomanes ", Psychologies, vol. 4, n ${ }^{\circ} 3$, p. 39-42.

FLATT, B. (1987). "Some stages of grief», Journal of Religion and Health, vol. 26, $\mathrm{n}^{\circ} 2$, p. 143-148.

FRÉCHETTE, L. (1997). «Deuil à la suite d'accidents de procréation et de décès de nourrissons », Frontières, vol. 9, n 3, p. 10-14.

FRÉCHETTE, L. (1988) «La mort dans les médias », Frontières, vol. 1, no 1, p. 17-21.

HANKINS, C. (1997). " Le sida et les comportements et situations à risque: Toxicomanie, incarcération et prostitution ", dans H. COHEN et J. LÉVY (dir.), Le sida : Aspects psychosociaux, culturels et éthiques, Montréal, Éditions du Méridien, p. 196-236.

HORWITZ, A. (1990). The Logic of Social Control, New York, Plenum Press, 290 p.

JACQUES, J. (1998). Psychologie de la mort et du deuil, Montréal, Éd. Modulo, 250 p.

KOLLAR, N. (1989). «Rituals and the disenfranchised grief », dans K.J. DOKA (dir.), Disenfranchised grief, New York, Lexington Books, p. 271-286.

LYNLEY, H.W. (2001). "Understanding workaholism : Data synthesis, theoretical critique and design strategies", International Journal of Stress Management, vol. 8, $\mathrm{n}^{\circ} 2$, p. 69-91.

MALMQUIST, C.P. (1986). "Children who witness parental murder: Posttraumatic aspects », dans Journal of American Academy of Child Psychiatry, vol. 25, n 3, p. 320-325.

MARIS, R.W. et al. (1992). Assessment and Prediction of Suicide, New York, Guilford, $697 \mathrm{p}$.

McCLEARY, R., K.S. CHEW, V. MERRILL et C. NAPOLITANO (2002). "Does legalized gambling elevates the risk of suicide? An analysis of U.S. counties and metropolitan areas ", Suicide and Life-Threatening Behavior, vol. 32, no 2, p. 209-221.

MILES, M.S. (1986). "Guilt in bereaved parents », dans T. RANDO (dir.), Parental Loss of a Child, Champaign, Illinois, Research Press Co., p. 97-118.

MONGEAU, S. (2002). "Lorsque la mort ne s'annonce pas. Le cas de la mort subite du nourrisson ", Frontières, vol. 14, n 2, p. 44-48.

PARKES, C.M. (1972). Bereavement: Studies of Grief in Adult Life, New York, International Universities Press, 233 p.
PETRY, N.M. et B.D. KILUK (2002). « Suicidal ideation and suicide attempts in treatment of pathological gamblers », Journal of Nervous and Mental Disease, vol. 190, n 7, p. 462-469.

RENO, Ronald A. (2000). Gambling's Impact on Families, <http://www.casinosno.org/ uploads/Gambling.htm>

ROSENBLATT, Paul C. (1988). «Grief: the social context of private feeling », Journal of Social Issues, vol. 44, n 3, p. 67-78.

ROOM, R. (1983). "Sociological aspects of the disease concept of alcoholism ", Research Advances in Alcohol and Drug Problems, sous la dir. de R. SMART et al., New York, Plenum Press, vol. 7, p. 47-91.

SÉGUIN, M., M.C. KIELY et A. LESAGE (1994). «L'après suicide, une expérience unique de deuil?", Santé mentale au Québec, vol. $11, \mathrm{n}^{\circ} 2$, p. 63-82.

SÉGUIN, M. et L. FRÉCHETTE (2001). Le deuil. Une souffrance à comprendre pour mieux intervenir, Montréal, Éd. Logiques, $249 \mathrm{p}$.

SÉGUIN, M. et J.A. SUISSA (2004). Liens entre suicide et jeu excessif: les problèmes associés au jeu excessif contribuent-ils au passage à l'acte?, Fonds québécois de recherche Science et Culture, Gouvernement du Québec (sous presse).

STROEBE, M.S. et W. STROEBE (1987). Bereavement and Health. The Psychological and Physical Consequences of Partner Loss, Cambridge, Cambridge University Press, $288 \mathrm{p}$.

SUISSA, J.A. (1998). Pourquoi l'alcoolisme n'est pas une maladie, Montréal, Fides, 191 p.

SUISSA, J.A. (2000). «Principes d'évaluation et d'intervention avec les familles ", dans Yves HURTUBISE et Jean-Pierre DESLAURIERS (dir.), Introduction au travail social, Québec, Presses de l'Université Laval, chap. 5, p. 117-151.

SUISSA, J.A. (2001). " Cannabis, social control and exclusion: The importance of social ties ", International Journal of Drug Policy, vol. 2, $\mathrm{n}^{\text {os }}$ 5-6, p. 385-396.

SUISSA, J.A. (2003). «A sociological perspective on gambling: A critical social analysis ", dans $D^{r}$ Nigel TURNER et Masood ZANGENEH (dir.), Psycho-structural Cybernetics of Problem Gambling, Canadian Mental Health and Addictions, Toronto University Press (sous presse).

TOURNIER, R. (1985). «The medicalization of alcoholism : Discontinuities in ideologies of deviance », Journal of Drug Issues, hiver, p. 39-49.

TOUSIGNANT, M. et T. PAYETTE (1997). Suicide et toxicomanie: deux phénomènes interreliés, Comité permanent de lutte à la toxicomanie, Gouvernement du Québec.

WEIL, A. (1983). From Chocolate to Morphine: Understanding Psycho Active Drugs, Boston, Houghton Mifflin, 240 p.

WEINBERG, N. (1994). «Self-blame, other blame and desire for revenge: Factors in recovery from bereavement », Death Studies, vol. $18, n^{\circ} 6$, p. 583-593. 